\title{
Early complications of endobronchial lung volume reduction treatment with endobronchial valves
}

\author{
(D) Deniz Doğan, (D) Cantürk Taşçı
}

University of Health Sciences Turkey, Gülhane Training and Research Hospital, Clinic of Chest Diseases, Ankara, Turkey

\section{Date submitted:}

13.04.2020

Date accepted:

07.05.2020

Online publication date: 15.06.2020

\section{Corresponding Author:}

Deniz Doğan MD, University of Health Sciences Turkey, Gülhane Training and Research Hospital, Clinic of Chest Diseases, Ankara, Turkey

dr_denizz@yahoo.com

ORCID:

orcid.org/0000-0003-2596-3113

Keywords: Endobronchial valve, emphysema, COPD, complication

\begin{abstract}
Aims: Endobronchial valve (EBV) treatment is one of the main minimally invasive treatment options for emphysema management. In this study, we aimed to evaluate the early term complication rates of patients having severe emphysema and being treated with EBV.

Methods: A total of fifteen patients treated with EBV for bronchoscopic lung volume reduction between November 2017 and January 2019 were included in the study. Pretreatment demographic data, pulmonary function results and exercise capacities were recorded. All complications encountered during the first 3-month period after the EBV treatment were evaluated. The status of collateral ventilation was assessed with Chartis system. Implanted valves were appropriately chosen according to the bronchial lumens' diameter. All patients were discharged after a minimum of 72 hours of observation.
\end{abstract}

Results: Fifteen bronchoscopic lung volume reduction treatment sessions were performed for fifteen patients. All of them were male. The mean age of the patients was $66.7 \pm 6.3$ years and the mean disease duration was $11.1 \pm 3.7$ years. A mean of 3.46 valves were implanted per patient. Early complications were observed in five patients (33.3\%) during the 3-month followup period after the EBV treatment. Of them, three had chronic obstructive pulmonary disease exacerbation, one had pneumothorax, and the last one had pneumonia. Only one patient died due to the early complications of the valve treatment.

Conclusions: Although the number of patients is small, our study demonstrates that EBV treatment can be safely applied with low complication rates as a bronchoscopic lung volume reduction treatment to the patients that have severe heterogeneous emphysema.

\section{Introduction}

Chronic obstructive pulmonary disease (COPD) is a widespread, preventable and treatable disease, characterized by restricted airflow and permanent respiratory tract symptoms originating from airway and/or alveolar abnormalities caused by severe exposure to harmful particles or gases (1). Emphysema, which constitutes the component of COPD other than chronic bronchitis, is a progressive condition characterized by irreversible damage to the alveolar tissue. As a result of permanent airway damage and early collapse in the small airways, air cannot be sufficiently expelled from the lungs in expirium and causes excessive lung ventilation.

The main cause of shortness of breath in patients with emphysema phenotype of COPD is this excess lung ventilation. The effect of current standard treatment approaches on excess lung ventilation is extremely limited. Although lung volume reduction surgery is an effective method in the treatment of emphysema, it is associated with a significant degree of morbidity (20-30\%) and increased early mortality (the first 3-month mortality) $(7.9 \%)$ (2). This has brought the use of bronchoscopic lung volume reduction treatments to the fore, as they have lower morbidity and mortality rates (3). Current studies in literature that have evaluated the efficacy of bronchoscopic lung volume reduction treatment methods have mostly been conducted on the use of endobronchial valves (EBV).

Reduction of hyperinflation of the lungs with EBV treatment is based on the principle of preventing entry in inspirium and drainage by allowing the air in the lungs with emphysema to be expelled in expirium. The aim of this study is to determine the early complications of EBV treatment in cases with advanced heterogenous emphysema. 


\section{Methods}

The study was approved by the Ethics Committee for the Non-invasive Research at the Gülhane University of Health Sciences Turkey (ID/date: 163/05.04.2019).

\section{Study design}

A retrospective analysis was made of patients that applied with EBV for bronchoscopic lung volume reduction treatment in our clinic between November 2017 and January 2019. Approval for the study was granted by the Ethics Committee of Health Sciences University Turkey, Gülhane Training and Research Hospital. The cases selected for treatment were those with Gold Stage 3 and 4 COPD with advanced emphysema. The treatment was applied with Zephyr® EBV (Pulmonx Inc, Redwood City, CA, USA).

To determine the distribution of emphysema, volumetric fine-slice thoracic tomography tests were used. Two target lung lobes were determined in cases who met the study selection criteria for bronchoscopic lung volume reduction treatment. In the determination of the first and second target lobes, quantitative lung perfusion scintigraphy was used. The lung lobes which had the lowest perfusion were determined as the target lobes. For patients who had received a sufficient duration of pulmonary rehabilitation, a record was made of demographic data such as age, gender, body mass index (BMI), duration of disease and smoking status before the procedure, and post-procedure respiratory function test parameters and exercise results were also recorded. Complications that developed in the follow-up period after the treatment of the patients that applied with EBV as bronchoscopic lung volume reduction treatment were also recorded.

\section{Patient selection criteria for EBV treatment}

1. Age $>18$ years

2. Heterogenous emphysema

3. GOLD Stage 3 or 4

4. Expected forced expiratory volume in one second (FEV1) $<50 \%$, residual volume $(\mathrm{RV})>150 \%$, total lung capacity $($ TLC) $>100 \%$

5. $\mathrm{RV} / \mathrm{TLC} \geq 58$

6. 6-minute walk test distance $<500 \mathrm{~m}$

7. Systolic pulmonary artery pressure $<50 \mathrm{mmHg}$ on echocardiography

8. Optimum use of bronchodilator treatment

9. Quitting smoking at least 8 weeks before the procedure

10. To have had pulmonary rehabilitation treatment for at least 6 weeks before the procedure

\section{Treatment procedure}

All the cases were hospitalized before the procedure. All the EBV placement procedures were applied in the bronchology unit of our clinic under intravenous total anesthesia, with a flexible bronchoscope (Olympus BF-1TQ180) having a working channel with a diameter of $2.8 \mathrm{~mm}$ passed through a laryngeal mask. After the selection of the target pulmonary lobes, whether or not there was collateral ventilation pre-procedure was evaluated with the Chartis system (PulmonX, Redwood City, CA, USA). In cases determined with collateral ventilation in the first target lobe, the Chartis procedure was repeated for the second target pulmonary lobe. The procedure was not applied to patients determined with collateral ventilation with the Chartis system.

In cases with negative Chartis, an EBV (Zephyr TM EBV; Pulmonx Inc., Redwood, CA, USA) was inserted unilaterally in the lobular, segmental or subsegmental bronchi, according to the anatomic status of the case, with the aim of completely isolating the target lobe. The inserted valves were selected to be compatible with airway diameters of 4.0-7.0 mm and 5.5-8.5 $\mathrm{mm}$. Following the procedure, routine postero-anterior chest X-ray was taken immediately in cases with symptoms, and after two hours for those without symptoms. Patients who were stable with no complications were monitored for 72 hours with daily chest $\mathrm{X}$-ray imaging and then discharged with a follow-up appointment at the end of the first week.

\section{Statistical Analysis}

Statistical Package for the Social Sciences (SPSS) for Mac 20.0 package program (SPSS Inc, Chicago, IL) was used for the statistical evaluation. Data were summarized as the mean and standard deviation for the continuous variables, as absolute value and percentages for the categorical variables.

\section{Results}

A total of fifteen cases were applied with fifteen bronchoscopic lung volume reduction treatment procedures. All the patients were male with a mean age of $66.7 \pm 6.3$ years and mean disease duration of $11.1 \pm 3.7$ years. A mean of 3.46 valves were placed per patient. The most common site of the procedure was the right superior lobe $(n=11)$. All the characteristics of the study population are shown in Table 1. The mean FEV1 was $0.71 \pm 0.24$ (0.35-1.35) L, and $26.5 \% \pm 8.6$ of the expected value, TLC was $7.12 \pm 0.76(6.27-8.41) \mathrm{L}$ and $117.1 \% \pm 15.4$ of the expected value, RV was $4.83 \pm 0.8$ (3.57-6.49) $\mathrm{L}$ and $204.9 \% \pm 38.3$ of the expected value, and the RV/TLC ratio was $68.4 \% \pm 6.6$. The mean BODE index (BMI, airflow obstruction, dyspnea, and exercise capacity) was $7.3 \pm 1.4$ and DLCO (diffusing capacity of the lung for carbon monoxide) was $35.1 \% \pm 12.4$ of the expected value. Complications were determined in five cases in the first 3-month period after the procedure.

Of these, COPD exacerbation was observed in three, pneumothorax in one, and pneumonia in one patient (Table 2). In all the cases who developed COPD exacerbation, the symptoms started in the second week. All these patients were re-hospitalized and treated with bronchodilator and systemic 


\begin{tabular}{|c|c|}
\hline Variable & Value $\pm S D /(\min .-\max )$. \\
\hline Age, year & $66.7 \pm 6.3$ \\
\hline BMI, $\mathrm{kg} / \mathrm{m}^{2}$ & $23.4 \pm 4.3$ \\
\hline Smoking history, pack-years & $42.5 \pm 6.2$ \\
\hline Disease duration, years & $11.1 \pm 3.7$ \\
\hline \multicolumn{2}{|l|}{ Lung functions } \\
\hline FVC, L & $2.27 \pm 0.6(1.5-3.3)$ \\
\hline FVC, \% predicted & $65.8 \pm 17(43-96)$ \\
\hline FEV1, L & $0.71 \pm 0.24(0.35-1.35)$ \\
\hline FEV1, \% predicted & $26.5 \pm 8.6(13-44)$ \\
\hline Total lung capacity, L & $7.12 \pm 0.76(6.27-8.41)$ \\
\hline Total lung capacity, $\%$ predicted & $117.1 \pm 15.4(101-155)$ \\
\hline Residual volume, L & $4.83 \pm 0.8(3.57-6.49)$ \\
\hline Residual volume, \% predicted & $204.9 \pm 38.3(155-281)$ \\
\hline DLCO, \% & $35.1 \pm 12.4(21-66)$ \\
\hline \multicolumn{2}{|l|}{ Arterial blood gas } \\
\hline $\mathrm{pCO}_{2}, \mathrm{mmHg}$ & $36.6 \pm 5.7$ \\
\hline $\mathrm{pO}_{2}, \mathrm{mmHg}$ & $67.4 \pm 15.7$ \\
\hline $\mathrm{sO}_{2}, \%$ & $91.9 \pm 5.0$ \\
\hline 6-minute-walk distance, $\mathrm{m}$ & $235.5 \pm 106.2(150-483)$ \\
\hline mMRC score* & $3.5 \pm 0.5$ \\
\hline CAT & $26.5 \pm 7$ \\
\hline BODE index score ${ }^{\dagger}$ & $7.3 \pm 1.4$ \\
\hline \multicolumn{2}{|l|}{ Target lobe, $n$} \\
\hline Right upper lobe & 11 \\
\hline Right upper + right middle lobe & 1 \\
\hline Right lower lobe & 1 \\
\hline Left upper lobe & 1 \\
\hline Left lower lobe & 1 \\
\hline Number of valves, per patient & $3.46 \pm 0.5$ \\
\hline $4.0 \mathrm{n}, \%$ & $25(48)$ \\
\hline 4.0 LP n, \% & $3(6)$ \\
\hline $5.5 \mathrm{n}, \%$ & $24(46)$ \\
\hline Total & 52 \\
\hline Hospital stay, days & $5.3 \pm 2.7(3-14)$ \\
\hline
\end{tabular}

SD: Standard deviation, FEV1: Forced expiratory volume in one second, FVC: Forced vital capacity, CAT: Chronic obstructive pulmonary disease assessment test, DLCO: Diffusing capacity of the lung for carbon monoxide, BMI: Body mass index, LP: Low product, BODE: Body mass index, airflow obstruction, dyspnea, and exercise capacity, mMRC: Modified Medical Research Council, Min.: Minimum, max.: Maximum. ${ }^{*} \mathrm{mMRC}$ dyspnea score scale ranges from 0 to 4 , with higher scores indicating more severe dyspnea.

$\mathrm{BODE}$ index score ranges from 0 to 10 based on a multidimensional scoring system to include FEV1, BMI, 6-minute-walk distance, and the $\mathrm{mMRC}$ dyspnea score. Higher scores denote a greater risk of mortality. steroid treatment in accordance with the GOLD guidelines (4). In one of the patients with COPD exacerbation, mortality developed 34 days after the procedure because of respiratory failure. The patient who developed pneumonia presented with complaints of shortness of breath, cough, expectoration of phlegm, and fever after two weeks. Three valves had been placed in the right lung superior lobe of this patient, but the pneumonia was in the right inferior lobe. No agent could be isolated in the mucous and blood cultures, and full resolution was achieved radiologically with appropriate antibiotherapy. In the case that developed pneumothorax, the complaints started 2 hours after the procedure in the form of sudden shortness of breath and chest pain. This patient was the only case where the bronchoscopic lung volume reduction procedure had been applied to the left lung superior lobe, and collateral ventilation was determined in the Chartis procedure in the right-side superior lobe of this patient. On the pulmonary radiograph taken on this patient, almost total leftsided pneumothorax was observed (Figure 1). Drainage was immediately applied by the Thoracic Surgery Department using a 32 French chest tube. Full expansion was obtained rapidly after tube thoracostomy. On the second day after the treatment,

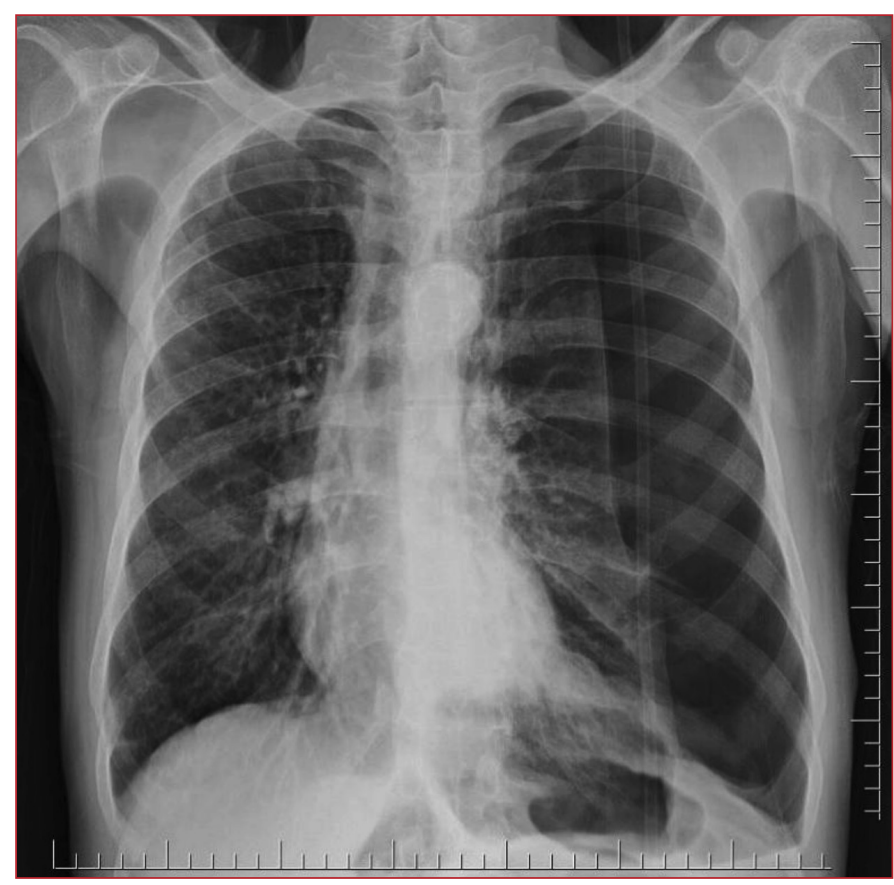

Figure 1. Left pneumothorax two hours after endobronchial valve procedure

\begin{tabular}{l}
$\begin{array}{l}\text { Table 2. Serious adverse events } \text { during } \mathbf{3} \text { months of } \\
\text { follow-up }\end{array}$ \\
\begin{tabular}{ll} 
Complication & $\mathbf{n}(\%)$ \\
\hline Pneumothorax & $1(6.7)$ \\
\hline Pneumonia & $1(6.7)$ \\
\hline COPD exacerbation & $3(20)$ \\
\hline Death & $1(6.7)$ \\
\hline COPD: Chronic obstructive pulmonary disease
\end{tabular} \\
\hline
\end{tabular}


air leakage and subcutaneous emphysema developed. When one week of air leakage had passed, it was planned to remove one of the valves, but on the $8^{\text {th }}$ day, the air leakage was seen to have stopped. The chest tube was removed on the $12^{\text {th }}$ day, and the patient was discharged on the 14th day. On the follow-up pulmonary radiograph taken after 4 weeks, total atelectasis was observed in the left superior lobe (Figure 2).

\section{Discussion}

In this study, complications and the frequency at which they developed were determined in the first three months after the application of bronchoscopic lung volume reduction treatment with endobronchial one-way valves to fifteen patients with advanced stage heterogenous emphysema. COPD exacerbation was observed in three patients, pneumonia in one and pneumothorax in one patient.

EBV treatment has been shown to provide significant functional and clinical improvement in patients with advanced stage heterogenous emphysema without collateral ventilation (5-8). In the first 3-month period after the placement of the valve, bronchitis, pneumonia and/or lung infection can develop at the rate of approximately $20 \%$ (9). In addition, the most frequently encountered complications related to valve treatment have been reported to be pneumothorax, pneumonia, COPD exacerbation, and valve migration $(10,11)$. Of these, pneumothorax is the most common complication which can develop (20-30\%) and is a clinical condition that requires attention (12).

Pneumothorax complication associated with the procedure is often $(86 \%)$ seen in the first 72 hours, so it is recommended that these patients are kept under observation for a minimum of 72

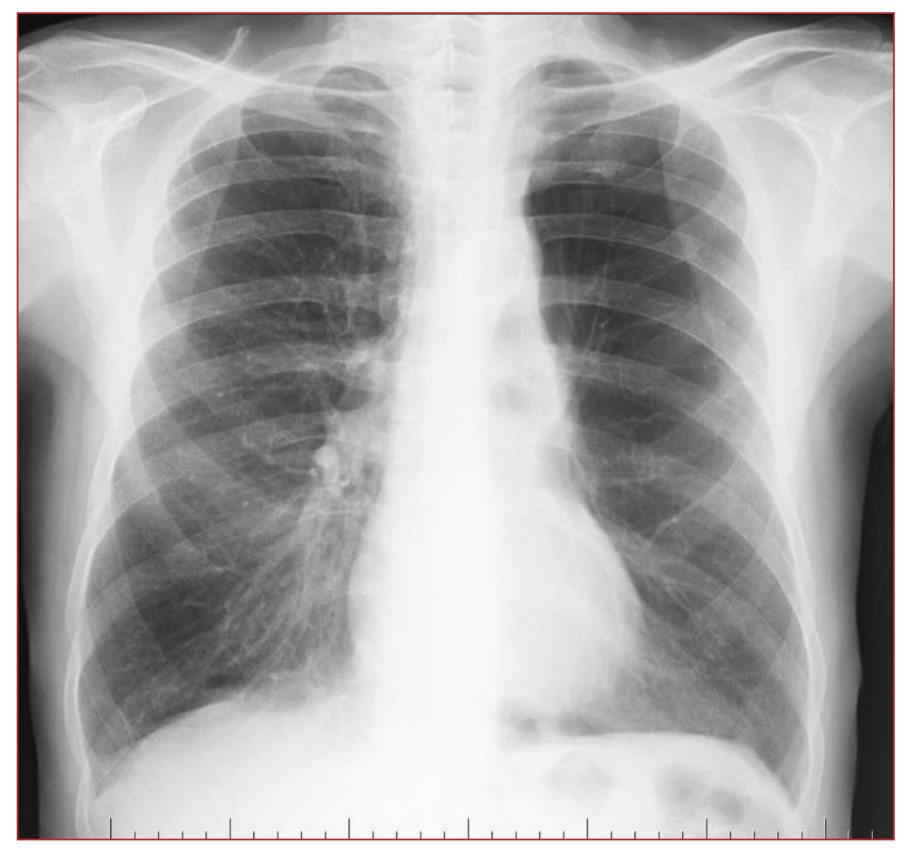

Figure 2. Total atelectasis in the left upper lobe four weeks after endobronchial valve procedure hours (13). In our clinical practice, patients are hospitalized and monitored for at least 72 hours after an EBV procedure. When examined in general, pneumothorax complication is seen more in-patient groups with a high treatment rate in the left superior lobe. For example, the highest pneumothorax rates have been reported as $29.2 \%$ in TRANSFORM studies and $26.6 \%$ in LIBERATE studies. The rates of right and left side superior lobe treatment were reported as $8 \%$ and $52 \%$, respectively, in the TRANSFORM study, and $6.3 \%$ and $66.4 \%$, respectively, in the LIBERATE study $(7,8)$.

In a study by Fiorelli et al. (14), 33 patients applied with a total of 36 procedures were followed up for 5 years, and despite left superior lobe treatment in $72 \%$, pneumothorax complication was seen in only $6 \%$. In the current study, pneumothorax developed in only 1 patient $(6.7 \%)$. The EBV treatment had been in the left superior lobe $(6.7 \%)$ and the case was treated with tube thoracostomy. On the second day of follow-up, there was seen to be air leakage and subcutaneous emphysema, then the air leakage stopped on the $8^{\text {th }}$ day. This patient was followed up for 12 days with tube thoracostomy, and the case had the longest hospitalization with a total of 14 days in this series.

In patients who develop pneumothorax associated with EBV treatment, when air leakage develops and persists for more than 7 days, it is recommended that one of the valves is removed, preferably the most proximal valve. If the air leakage continues for more than 48 hours despite the removal of one valve, then all the valves must be removed (15). In the current case, as the air leakage stopped on the 8th day, there was no need to remove a valve.

Another frequently seen complication associated with EBV treatment is COPD exacerbation. In the LIBERATE study which was the first, prospective, randomized, controlled study conducted to evaluate the efficacy and reliability of Zephyr® EBV, a total of 190 patients with minimal or no collateral ventilation (128 EBV, 62 standard medical treatment) were evaluated over a 12-month period. The development of COPD exacerbation was reported at the rate of $7.8 \%$ in the first 45 days after treatment and at $23 \%$ in the subsequent period (8). The highest rate of COPD exacerbation was reported at the rate of $37.8 \%$ in the European arm of the VENT study (9). In the current study, COPD exacerbation developed in 3 patients $(20 \%)$, all within the first 2 weeks. In literature, the mortality rate of patients applied with EBV treatment ranges from $0 \%$ to $8 \%(16,17)$. In the current study, of the three patients with COPD exacerbation, mortality occurred in one $(7.6 \%)$ because of respiratory failure 34 days after the treatment.

The rate of pneumonia development in the early period after EBV treatment has been reported as $1.2-18 \%$ in previous studies in literature (14). In the current study, pneumonia developed in the right inferior lobe in one patient two weeks after the treatment. However, the EBV treatment had been applied to the right superior lobe in this patient. No agent could be isolated in this 
patient, who was then hospitalized and treated with a diagnosis of community-acquired pneumonia. Following the treatment, full resolution was achieved clinically and radiologically. When pneumonia develops in the pulmonary lobe where the valve has been applied, the valve must be removed. However, in the case in this study, as pneumonia developed in a different pulmonary area, it was not deemed necessary to remove the valve.

The main limitation of this study was the low number of cases. However, it can be considered that, with an increased number of cases and range of procedures, the complication rate could be lower.

\section{Conclusion}

In conclusion, EBV treatment applied for bronchoscopic lung volume reduction treatment to suitable patients with advanced stage emphysema is a method which can be performed with a low rate of complications.

\section{Ethics}

Ethics Committee Approval: The study was approved by the Ethics Committee for the Non-invasive Research at the Gülhane University of Health Sciences Turkey (ID/date: 163/05.04.2019).

Informed Consent: Retrospective study.

Peer-review: Externally and internally peer-reviewed.

\section{Authorship Contributions}

Surgical and Medical Practices: D.D., C.T., Concept: D.D., C.T., Design: C.T., Data Collection or Processing: D.D., Analysis or Interpretation: D.D., C.T., Literature Search: D.D., C.T., Writing: D.D., C.T.

Conflict of Interest: No conflict of interest was declared by the authors.

Financial Disclosure: The authors declared that this study received no financial support.

\section{References}

1. Global Strategy for the Diagnosis, Management, and Prevention of Chronic Obstructive Pulmonary Disease 2019 Report. Global Initiative for Chronic Obstructive Lung Disease (GOLD). http://www.goldcopd.org

2. Fishman A, Martinez F, Naunheim K, et al. A randomized trial comparing lung-volume-reduction surgery with medical therapy for severe emphysema. N Engl J Med. 2003;348:2059-2073.

3. Flandes J, Soto FJ, Cordovilla R, Cases E, Alfayate J. Bronchoscopic Lung Volume Reduction. Clin Chest Med. 2018;39:169-180.

4. Vestbo J, Hurd SS, Agustí AG, et al. Global strategy for the diagnosis, management, and prevention of chronic obstructive pulmonary disease: GOLD executive summary. Am J Respir Crit Care Med. 2013;187:347-365.

5. Sciurba FC, Ernst A, Herth FJ, et al. A randomized study of endobronchial valves for advanced emphysema. N Engl J Med. 2010;363:1233-1244.

6. Klooster K, Hartman JE, ten Hacken NH, Slebos DJ. One-year follow-up after endobronchial valve treatment in patients with emphysema without collateral ventilation treated in the STELVIO trial. Respiration. 2017;93:112-121.

7. V Kemp SV, Slebos DJ, Kirk A, et al. A multicenter randomized controlled trial of Zephyr endobronchial valve treatment in heterogeneous emphysema (TRANSFORM). Am J Respir Crit Care Med. 2017;196:1535-1543.

8. J. Criner G, Sue R, Wright S, et al. A Multicenter Randomized Controlled Trial of Zephyr Endobronchial Valve Treatment in Heterogeneous Emphysema (LIBERATE). Am J Respir Crit Care Med. 2018;198:1151-1164.

9. Herth FJ, Noppen M, Valipour A, et al. Efficacy predictors of lung volume reduction with Zephyr valves in a European cohort. Eur Respir J. 2012;39:1334-1342.

10. Herth FJ, Eberhardt R, Gompelmann D, et al. Radiological and clinical outcomes of using Chartis to plan endobronchial valve treatment. Eur Respir J. 2013;41:302-308.

11. Slebos D, Shah PL, Herth FJ, Valipour A. Endobronchial Valves for Endoscopic Lung Volume Reduction: Best Practice Recommendations from Expert Panel on Endoscopic Lung Volume Reduction. Respiration. 2017;93:138-150.

12. Klooster $\mathrm{K}$, ten Hacken $\mathrm{NH}$, Hartman JE, Kerstjens HA, van Rikxoort EM, Slebos DJ. Endobronchial valves for emphysema without interlobar collateral ventilation. N Engl J Med. 2015;373:2325-2335.

13. Gompelmann D, Benjamin N, Kontogianni K, et al. Clinical and radiological outcome following pneumothorax after endoscopic lung volume reduction with valves. Int J Chron Obstruct Pulmon Dis. 2016;11:3093-3099.

14. Fiorelli A, Santoriello C, De Felice A, et al. Bronchoscopic lung volume reduction with endobronchial valves for heterogeneous emphysema: long-term results. J Vis Surg 2017;3:170.

15. Valipour A, Slebos DJ, de Oliveira HG, et al. Expert Statement: Pneumothorax Associated with Endoscopic Valve Therapy for Emphysema--Potential Mechanisms, Treatment Algorithm, and Case Examples. Respiration. 2014;87:513-521.

16. Valipour A, Slebos DJ, Herth F, et al. Endobronchial valve therapy in patients with homogeneous emphysema. Results from the IMPACT study. Am J Respir Crit Care Med. 2016;194:1073-1082.

17. Davey C, Zoumot Z, Jordan S, et al. Bronchoscopic lung volume reduction with endobronchial valves for patients with heterogeneous emphysema and intact interlobar fissures (the BeLieVeR-HIFi study): a randomized controlled trial. Lancet 2015;386:1066-1073. 